In 1982 we started the project 'Cryogenic Magnetometers' with the aim to develop SQUID-magnetometers appropriate to a large variety of applications. The first system we developed is a SQUIDmagnetometer with an open-ended horizontal access at room temperature. The measuring space inside the pick-up coils is easy accessible and the magnetization (remanent or induced) can be measured of materials in a static position or transported through the access.

Further, systems have been developed with the pick-up coil set outside the cryostat, in order to arrive at a flexible system with which the object under investigation can be approached as close as possible. Another part of the project is the optimization of magnetometers with respect to the cryogenic system in order to develop cryogenic magnetometer systems with a very long time between helium refills. The present status of these three research subjects is briefly described.

\title{
Cryogenic magnetometer research at Twente University of Technology
}

\author{
H.J.M. ter Brake and J. Flokstra
}

Keywords: cryogenics, magnetometer, research

Magnetometry has been one of the main research subjects of our low temperature group. For a decade we have developed SQUID-magnetometer systems for biomagnetic measurements ${ }^{1}$ and for the study of the dynamic behaviour of magnetic materials. ${ }^{2}$ In 1982 we started the 'Cryogenic Magnetometers' project with the aim of developing SQUID-magnetometers appropriate to a large variety of applications.

The first system we developed was a SQUIDmagnetometer with an open-ended horizontal access at room-temperature. The measuring space inside the pick-up coils of the SQUID-system is easy accessible and the magnetization (remanent or induced) of materials in a static position or transported through the access can be measured.

Systems have also been developed with the pickup coil set outside the cryostat in order to arrive at a flexible system with which the object under investigation can be approached as closely as possible.

Another part of the project is the optimization of magnetometers with respect to the cryogenic system. The final aim is to develop cryogenic magnetometer systems with a very long time between helium refills

In this paper the present status of these three research subjects is briefly described.

\section{SQUID-magnetometer with horizontal room-temperature access}

Our SQUID-magnetometer with horizontal access cryostat is presented in Fig. 1. This prototype cryostat (height $78 \mathrm{~cm}$, diameter $36 \mathrm{~cm}$, helium reservoir $18 \mathrm{l}$, boil-off rate $0.18 \mathrm{l} \mathrm{h}^{-1}$ ) is equipped with two vapourcooled copper radiation shields $(180$ and $80 \mathrm{~K})$ and an access pipe with an inner diameter of $10 \mathrm{~mm}$. Characteristic of this instrument is that the super- conducting elements are not cooled by direct contact with liquid helium, but that cooling is obtained via thermal conduction realized by rigidly mounting these elements to the copper bottom plate of the cryostat. The cryogenic system can be easily dismantled so that modification of and repairs to the sensing systems can be rapidly performed.

In order to detect the magnetic flux applied in the access we use one pick-up coil consisting of 4 turns (diameter $40 \mathrm{~mm}$ ) directly connected to the SHESQUID. Magnetic noise from the surroundings is suppressed by means of a lead cylinder, made of thin lead foil wrapped once around a copper cylinder holder (Fig. 1). The noise reduction for homogeneous disturbing fields amounts to $7.2 \times 10^{-6}$. The copper holder also supports a single-layer coil for the application of static magnetic fields. Fields up to $12 \mathrm{mT}$ can be trapped by the lead cylinder.

The noise level and the dynamic behaviour of the system are determined by the thermal noise and eddy currents in the copper elements. With respect to a magnetic dipole input, the noise level is $3 \times 10^{-11} \mathrm{Am}^{2}$ $(\mathrm{Hz})^{-1 / 2}$. The dynamic behaviour can be characterized by a low-pass frequency response having a $-3 \mathrm{~dB}$ point at $20 \mathrm{~Hz}$.

We used the SQUID-magnetometer for measuring remanent magnetization of rock samples (rock magnetometer application) and for measuring induced magnetization (susceptometer application). A detailed description of these successful test experiments will be published elsewhere. ${ }^{3}$ Here we present in Fig. 2 the results for the detection of the oxygen concentration in expired air transported through the access (oxygen is the dominant paramagnetic component in air). It is a good demonstration of one of the features of this instrument, ie measuring instantaneously the magnetic concentration in flowing materials. 


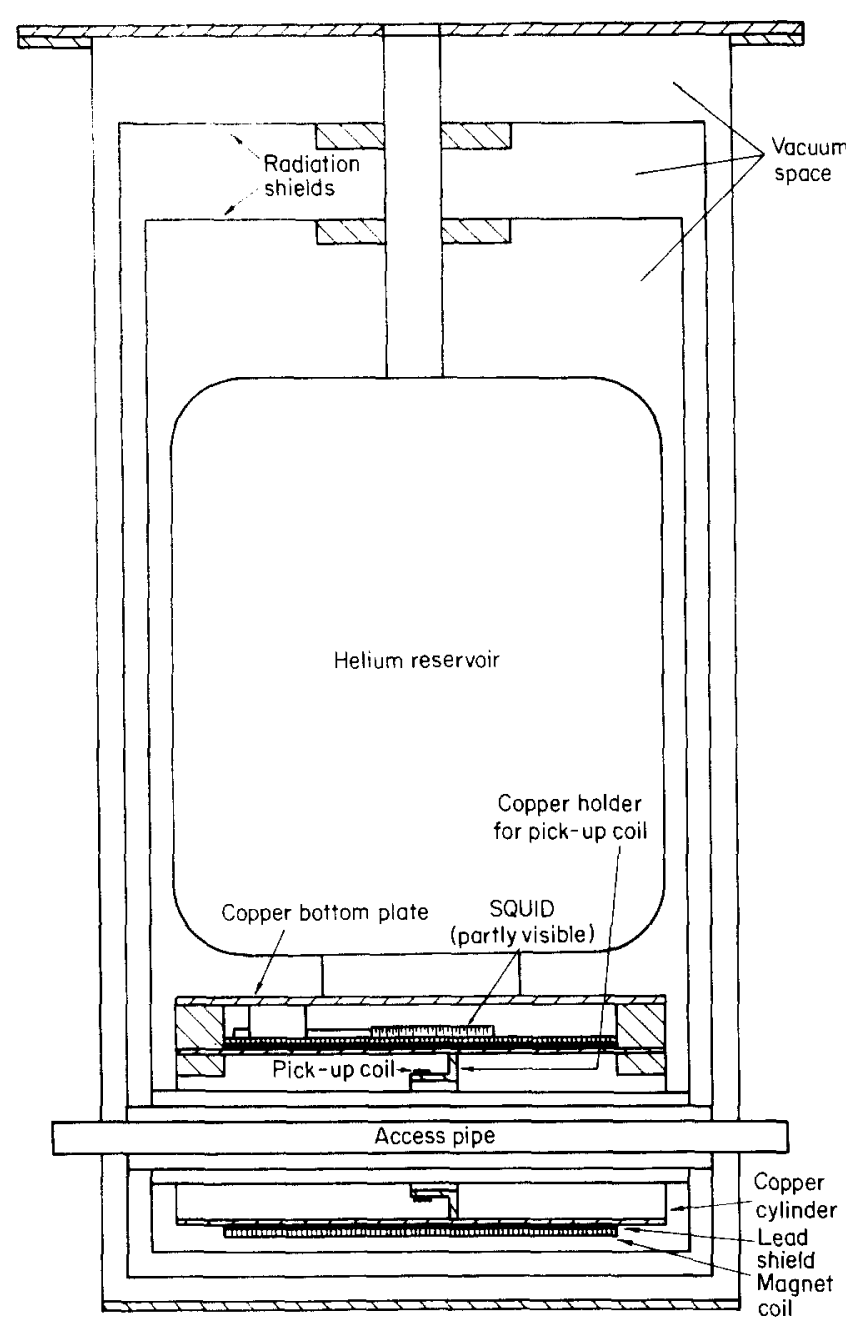

Fig. 1 Horizontal-access cryostat

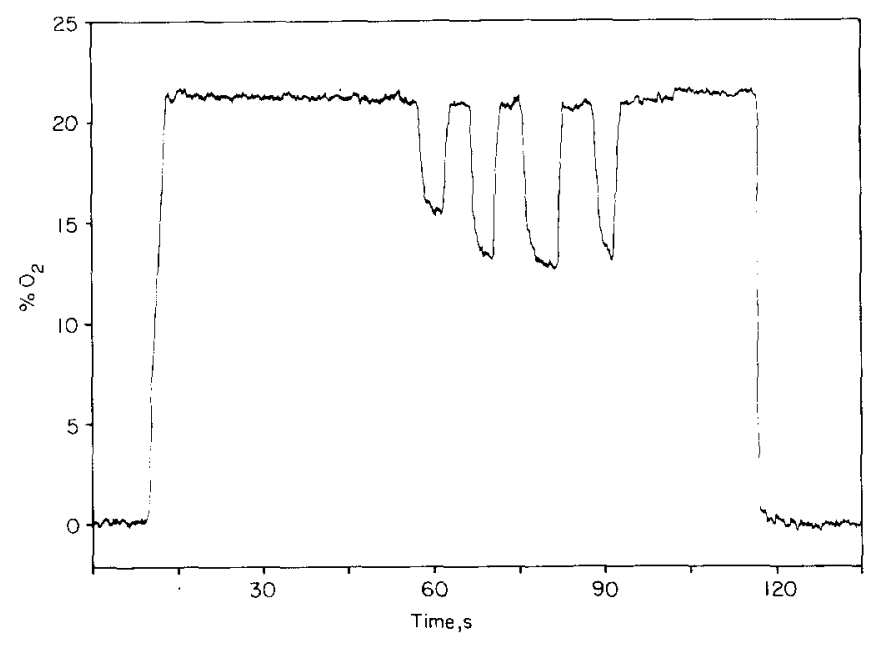

Fig. 2 Registration of the oxygen concentration in the access in expired air

\section{SQUID-magnetometer with room- temperature pick-up coils}

Flexibility and accessibility of the pick-up coil system can be improved by placing this part of the flux-transformer circuit outside the cryostat at the expense of an increase in the noise level and a highpass frequency response due to the normal resistance of pick-up coils and connecting leads. It is necessary to use a large self-inductance in order to limit the characteristic frequency of this high-pass behaviour and this was realized by applying a transformer between pick-up coils and SQUID in such a way that optimal signal transfer was also obtained.

This principle of external flux coupling has been applied by Cerdonio et $\mathrm{al}^{4}$ for the construction of sensitive current meters and by Wikswo ${ }^{5}$ for measuring the current through a nerve using a Rogowski-type sensing coil. Our research concerns the use of open pick-up coils and therefore the suppression of ambient noise is more relevant than for the systems of Cerdonio and Wikswo.

Under conditions of very low external noise the sensitivity of the system is determined by the thermal noise of the input circuit. For this case we have developed a magnetometer with an astatic pick-up coil set of 2 times 200 turns (inner diameter $40 \mathrm{~mm}$ ) and a transformer consisting of 925 primary and 2 secondary turns wound around a toroidal shaped cryoperm core (inner diameter $16 \mathrm{~mm}$, outer diameter $20 \mathrm{~mm}$ ). The instrument has a cut-off frequency of $18 \mathrm{~Hz}$ and a noise level of $4.7 \times 10^{-10} / f \mathrm{~T}(\mathrm{~Hz})^{-1 / 2}$ (f being the signal frequency). An improved version will be applied in our biomagnetic research programme.

In the case of a high external noise level, it is necessary to use a pick-up coil of which the dipole sensitivity decreases rapidly with increasing distance. We have developed a simple sensing array of four coils with a dipole sensitivity proportional to the eighth inverse power of the distance $r$. Design considerations of such pick-up coils and test experiments of this $r^{-8}$ coil are described in a separate paper. ${ }^{6}$

\section{Cryogenic developments}

Conventional SQUID-magnetometers are cooled with liquid helium. Because not all the potential users have liquid helium facilities at their disposal this severely hampers large scale application of SQUIDmagnetometers. Therefore, the development of more or less stand-alone systems is relevant. One of the possibilities in this respect is the use of a simple closed-cycle refrigerator in combination with a liquidhelium bath in order to obtain a system with a very long time between helium refills. Our work concerns the optimization of such a combination.

We have constructed a cryostat, bath volume 101 , equipped with a R 210 refrigerator of Leybold Hereaus (cooling power $1 \mathrm{~W}$ at $15 \mathrm{~K}$ ) cooling the two radiation shields. The boil-off rate in this combination is $2 \times 10^{-3} \mathrm{l} \mathrm{h}^{-1}$. resulting in an operation time of more than 200 days. Details of this system will be described elsewhere?

\section{Further developments}

We are now working on a magnetometer having a horizontal access with a diameter of $38 \mathrm{~mm}$. The dipole sensitivity of this magnetometer will be $10^{-11} \mathrm{Am}^{2}(\mathrm{~Hz})^{-1 / 2}$ and the magnetization can be sensed in three perpendicular directions. The magnetometer will be used as rock magnetometer for paleomagnetic research.

The applicability of magnetometer systems with external flux coupling is further studied. Especially the measurement of small displacements has our attention. 
In this case a small magnetic particle is attached to the moving material.

The optimization of cryogenic systems will be further developed using a LSI 11/73 for computer aided cryostat design.

\section{Authors}

The authors are from Twente University of Technology, Department of Applied Physics, POB 217, 7500 AE Enschede, The Netherlands. Paper received May 1984.

This work is part of the research programme of the Foundation for Fundamental Research on Matter (FOM) and was made possible by financial support from the Netherlands Organization for the Advancement of Pure Research (ZWO).

\section{References}

1 Peters, M.J., Dunajski, Z., Heringa, A. and van Dam, R.Th. Il Nuovo Cimento $2 D$ (1983) 311

2 Overweg, J.A., ter Brake, H.J.M., Flokstra, J. and Gerritsma, G.J. J Phys E 16 (1983) 1247

3 ter Brake, H.J.M., Ulfman, J.A. and Flokstra, J. SQUIDmagnetometer with open-ended horizontal room-temperature access, to be published in $J$ Phys $E$

4 Cerdonio, M., Ricci, F.F. and Romani, G.L. J Appl Phys 48 (1977) 4799

$5 \quad$ Wikswo Jr., J.P. J Appl Phys 52 (1981) 2554

6 Kuper, F.G., ter Brake, H.J.M. and Flokstra, J. Magnetometer sensing coils with extreme signal-to-interference discrimination, contribution to LT 17, Karlsruhe, (1984)

7 ter Brake, H.J.M., Hogenkamp, J.E.M., Ulfman, J.A. and Flokstra, J. Low evaporation hybrid cryogenic system for superconducting devices, ICEC 10 Proceedings Butterworths, UK (1984) (to be published) 